\title{
Leonardo Corona: dal «bozzetto » all'opera definitiva? Storia, funzione e statuto del monocromo con i santi Agostino, Monica, Nicola da Tolentino e Gugliemo d'Aquitania della chiesa di Santo Stefano in Venezia
}

Valentina Sapienza

Il visitatore che decida di varcare il portale della chiesa di Santo Stefano rimane innanzitutto colpito dall'imponente soffitto ligneo a carena di nave, decorato da rosoni e motivi floreali dipinti. Qualche istante dopo, si precipita solitamente alla ricerca più o meno affannosa dei Tintoretto ${ }^{\mathrm{I}}$ provenienti dalla chiesa di Santa Margherita e attualmente custoditi in sacrestia. I più informati non mancano di rendere omaggio al sofisticato Battesimo di Cristo, attribuito a Pomponio Amalteo, che decora l'altar maggiore del battistero, o ancora alla Stele funeraria di Giovanni Falier realizzata da Canova nel i808.

In realtà, innumerevoli sono i capolavori che le alte mura della chiesa dei Padri Eremitani di Sant'Agostino custodiscono gelosamente da molti secoli. E tra di essi, ci dedicheremo in questa occasione a una delle meno conosciute, eppure più curiose testimonianze artistiche conservate in situ. Mi riferisco al dipinto su tavola (fig. I), letteralmente appeso contro una delle pareti della cappella destra del transetto dedicata a Sant'Agostino, che solo in pochi hanno avuto la fortuna di osservare da vicino ${ }^{2}$.

ASVe : Archivio di Stato di Venezia. ASPV : Archivio Storico del Patriarcato di Venezia. I dipinti del Robusti eseguiti per la Scuola del Santissimo Sacramento della chiesa di Santa Margherita raffigurano rispettivamente un'Ultima cena, una Lavanda dei piedi e un' Orazione nell'orto.

2 L'ingresso alla cappella è tassativamente proibito perfino agli studiosi con motivate necessità di accesso. Occorre rivolgersi all'apposito ufficio della Curia Patriarcale di Venezia per ottenere una sorta di lasciapassare. Va inoltre precisato che le condizioni di luce non sono sempre ottimali e conviene optare per una visita mattutina, intorno alle I0.30, possibilmente in un bella giornata soleggiata. 
La tavola ${ }^{3}$, interamente a monocromo, è opera del misconosciuto pittore muranese Leonardo Corona ${ }^{4}$. Essa raffigura la porzione sottostante della pala d'altare dello stesso autore con la Madonna della cintura con i santi Agostino, Monica, Stefano, Nicola da Tolentino e Guglielmo d'Aquitania (fig. 2), che decora ancor oggi il primo altare della navata sinistra della nostra chiesa.

Della misteriosa tavola, saltata fuori per caso nel corso di una campagna di restauri condotta sotto la direzione di Francesco Valcanover nel 197I, non sappiamo praticamente nulla. Rimossa in quell'occcasione la tela dall'altare per provvedere al restauro delle parti marmoree e alla pulitura del dipinto, i restauratori si sono trovati di fronte a uno "spettacolo» inusuale (fig. 3 ) : al posto del tradizionale tavolato in legno, dietro la tela è apparso il monocromo, ivi collocato - si è detto - « a rinforzo " della stessa'. E su questo punto torneremo.

Ma da dove viene questo oggetto? Che funzione aveva? E per entrare nel vivo del dibattito di cui questo convegno intende occuparsi, che tipo di rapporto possiamo ipotizzare abbia intrattenuto con il dipinto che decora attualmente l'altare?

Sul monocromo si segnalano i seguenti contributi bibliografici : Antonio Niero, Chiesa di Santo Stefano in Venezia, Padova, 1978, p. 74; Giovanna Nepi Scirè, "Madonna della cintola e santi ", in Da Tiziano a el Greco. Per la storia del Manierismo a Venezia I540-I59o, Rodolfo Pallucchini (a cura di), cat. esp., Venezia, Palazzo Ducale, 198I, Milano, I98I, p. 228-230 (con bibl.); Maria Agnese Chiari Moretto Wiel, Andrea Gallo, Ettore Merkel, Chiesa di Santo Stefano. Arte e e devozione, Venezia, 1996, p. 25-28; William Roger Rearick, Il disegno veneziano del Cinquecento, Milano, 200I, p. I97-I98; Cristina Bragaglia, «I santi Agostino, Monica, Nicola da Tolentino, Guglielmo d'Aquitania e angeli ", in Centro Studi Agostino Trapè di Tolentino. Comitato Nazionale VII Centenario di san Nicola. San Nicola da Tolentino nell'arte. Corpus iconografico, I. Dalle origini al Concilio di Trento; II. Dal Concilio di Trento alla fine del Seicento, Roberto Tollo (a cura di), coordinamento scientifico di Valentino Pace con la collaborazione di Mario Marubbi, Tolentino, Biblioteca Egidiana, 2006, II, n. 34, p. 243 (con bibl.).

Leonardo Corona nasce a Murano nel 1552 circa e muore a Venezia a soli quarantaquattro anni il I7 ottobre I596. Nonostante oggi siano in pochi conoscere il suo nome, si tratta di uno dei più abili pittori attivi a Venezia e dintorni nella seconda metà del XVI secolo. Non a caso Boschini lo classifica secondo per merito, subito dopo Palma il Giovane, tra i pittori delle Sette Maniere. Se si eccettuano le notizie tramandate da Ridolfi e qualche raro articolo ormai datato, l'opera di questo pittore, attivo nei più importanti cantieri veneziani di fine secolo (da Palazzo Ducale, alla chiesa di San Giuliano, da San Nicolò de’ Mendicoli a San Giovanni in Bragora, dalla Scuola de' Picai a San Fantin, a San Giovanni Elemosinario), non ha mai ricevuto l'attenzione che merita. Ecco le ragioni che mi hanno spinto a scegliere di dedicare a lui la mia tesi di Dottorato. Per la ricostruzione del profilo biografico di Corona si veda Valentina Sapienza, «Leonardo Corona, 1552-1596 ", Venezia Cinquecento, XVI (luglio-dic. 2006), 32, p. 195-207. Per qualche spunto bibliografico su Corona vedi ivi, p. 205, nota 4.

$5 \quad$ Così recita la scheda OA (Opera d'Arte) n. I892I4 della Soprintendenza per i beni storico-artistici e demo, etnoantropologici di Venezia, a cura di F. Colombo e aggiornata da L. Levolella. Mi spiace segnalare che l'autore della scheda, complice anche chi ha provveduto all'aggiornamento, ha compiuto diversi errori : innanzitutto l'attribuzione della tavola a tale Matteo (sic) Pagani, pittore attivo tra la fine del Seicento e l'inizio del secolo successivo nel centro Italia. L'errore attributivo si deve al fatto che Antonio Niero nella già menzionata guida dedicata alla chiesa di Santo Stefano ipotizza che la tavola debba identificarsi con il dipinto del veneziano Paolo Pagani, citato da Tassini presso la Scuola dei Centurati. Si vedano : Giuseppe Tassini, Edifici di Venezia distrutti o volti ad uso diverso da quello a cui furono in origine destinati, Ia ed. Venezia, I885, ed. cons. Venezia, 1969, p. 66; A. Niero, Chiesa di Santo Stefano..., op. cit. nota 3, p. 74. Un' ipotesi, quella di Niero, che in ogni caso va respinta : Tassini parla di un dipinto finito di tutto punto e non di un monocromo appena abbozzato, e in ogni caso la nostra tavola non può datarsi alla seconda metà del XVII secolo. Quanto alla funzione di « rinforzo » che essa avrebbe avuto, posta com'era dietro la tela definitiva, avrò più avanti l'opportunità di chiarire la mia posizione. 


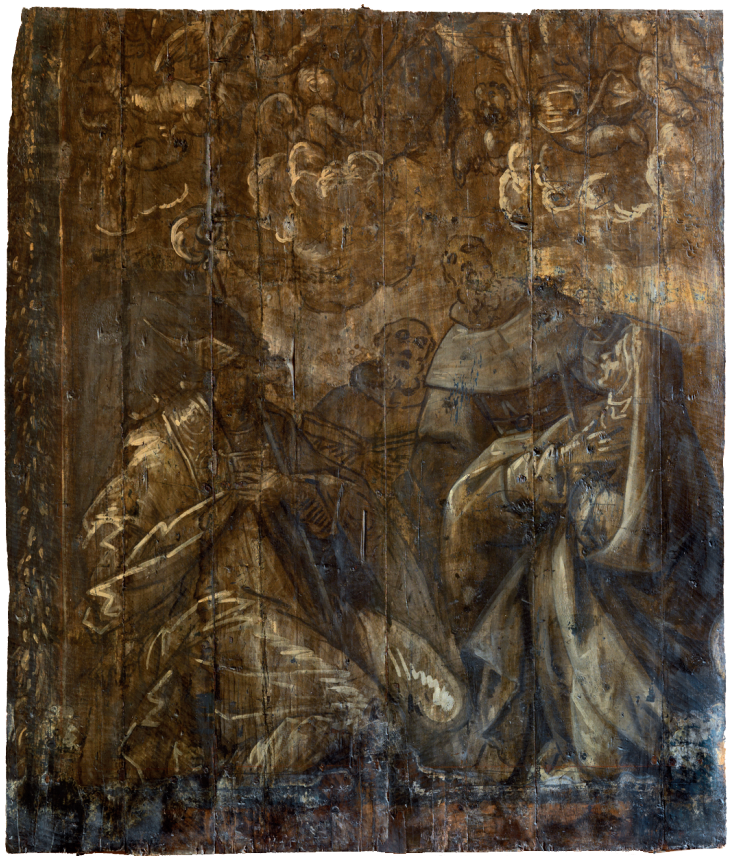

Fig. 1 - Leonardo Corona, Les saints Augustin, Monique, Nicolas de Tolentino et Guillaume d'Aquitaine, I590, Venise, église de Santo Stefano (C) Cameraphoto, Venise.

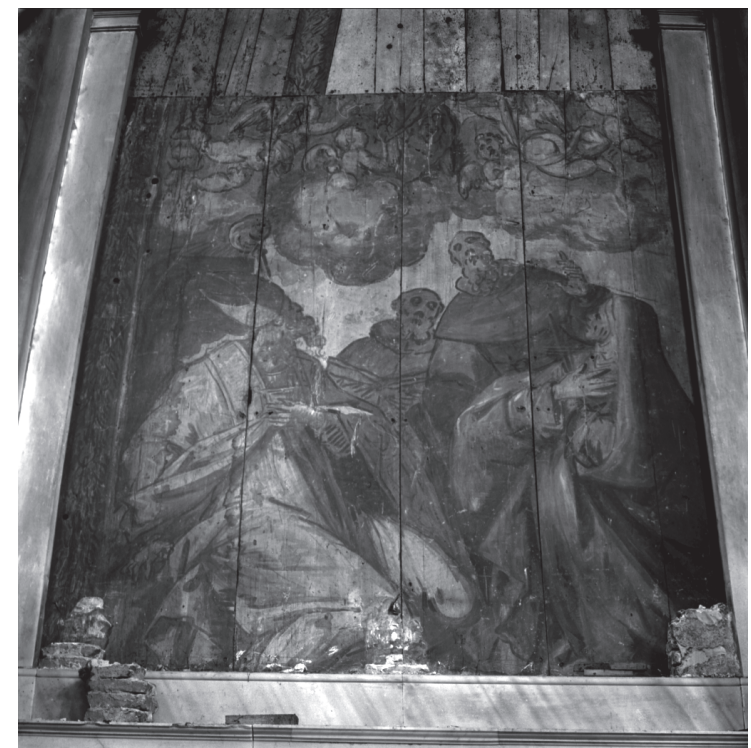

Fig. 3 - Leonardo Corona, Les saints Augustin, Monique, Nicolas de Tolentino et Guillaume d'Aquitaine, 1590, Venise, église de Santo Stefano (C) Soprintendenza speciale per il Polo Museale, Venise.

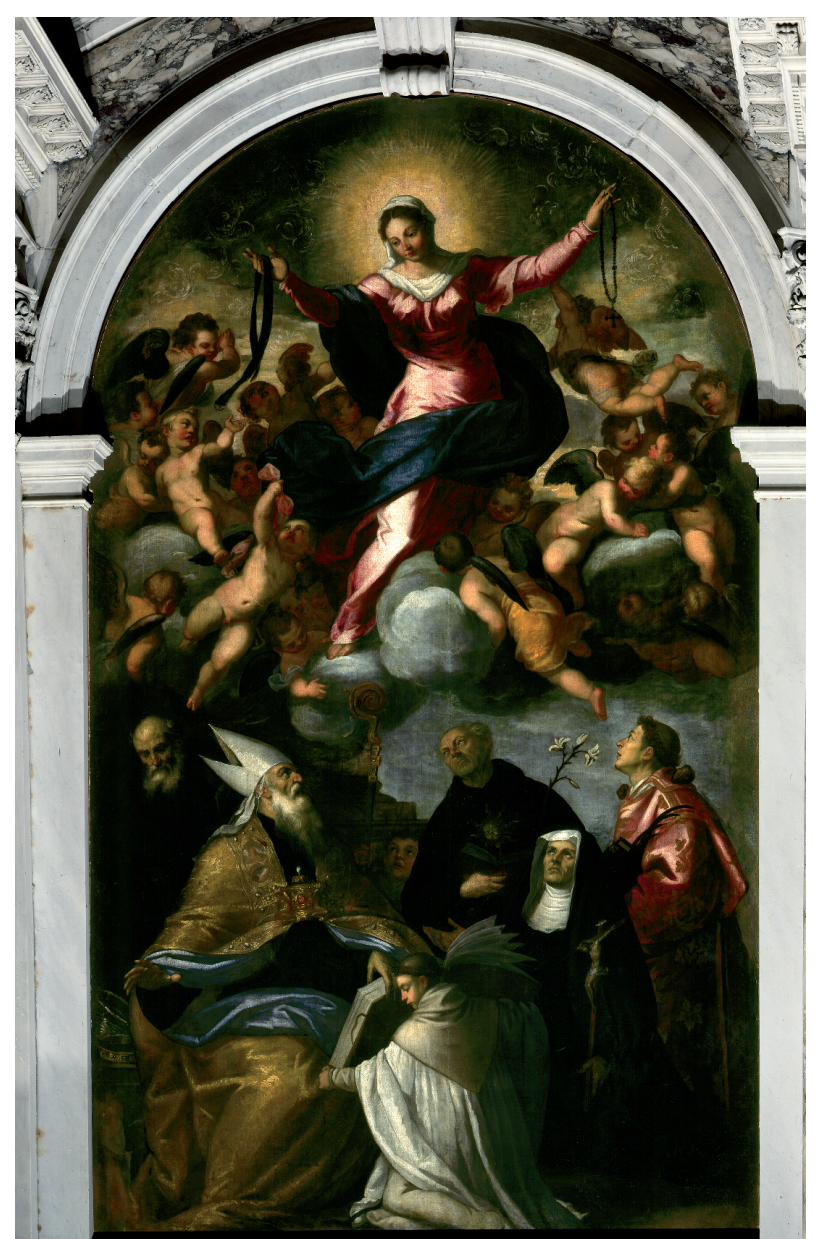

Fig. 2 - Leonardo Corona, La Vierge à la ceinture avec les saints Augustin, Monique, Nicolas de Tolentino et Guillaume d'Aquitaine, I59I-92, Venise, église de Santo Stefano (C) Cameraphoto, Venise. 
Per rispondere a questi e ad altri interrogativi sarà necessario innanzitutto approfondire il contesto per cui monocromo e tela sono stati concepiti, successivamente analizzare la natura materiale del "bozzetto " (le virgolette saranno chiarite al momento opportuno) e i cambiamenti iconografici presenti nell'opera definitiva.

\section{Un po' di contesto}

Il 6 febbraio 1590, la Scuola dei Centurati congregatasi sotto l'invocazione di Santa Maria della Consolazione e la protezione dei santi Agostino e Monica, e in unione con l'omonima confraternita della chiesa di San Giacomo di Bologna, ottiene la concessione del primo altare a sinistra della chiesa di Santo Stefano a Venezia ${ }^{6}$. La confraternita esisteva in realtà da quasi un decennio, se già Nicolai ${ }^{7}$ precisa che i Centurati, riunitisi per la prima volta nel I58I, l'anno seguente organizzavano una bella processione con tanto di suonatori, cui partecipavano anche alcuni religiosi del convento ${ }^{8}$. Nell'ottobre 1583 la Scuola avanza una serie di richieste ai frati, abbozzando una prima versione dello statuto in attesa della compilazione ufficiale della Mariegola9. Il documento inedito costituisce, a mia conoscenza, la prima testimonianza dell'esistenza della confraternita e ci permette di comprendere assai agilmente la natura particolare di questa scuola di devozione :

I583 mensis octobris / Havendo noi fratelli della Compagnia della Cintura col nome di Dio, più volte congregatj per ben fondare, stabilire et ordinare ditta compagnia, habbiamo dal corporal di

ASVe, Provveditori di Comun, reg. V, « Matricola della Scola della Beata Vergine della Centura in S. Stefano ", c. 445. L'informazione si ricava da una parte più tarda, datata I4 marzo I597, in cui la Scuola chiede conferma ai Padri Eremitani della concessione dello spazio posto innanzi al proprio altare, per la costruzione delle sepolture per i confratelli e le consorelle. Sebbene la concessione fosse già contemplata all'epoca dell'accordo riguardante l'altare (per questo se ne evoca la data), i confratelli non vogliono in alcun modo generare contrasti con i religiosi e non mancano allora di chiedere ulteriore conferma.

7 Agostino Nicolai, Memoria manoscritta sopra la chiesa e di Santo Stefano, Venezia, Biblioteca civica del museo Correr, ms. Cic. I877. Si tratta di una delle fonti più preziose sulla storia del convento. Essa risale al XVIII secolo ed è stata compilata sulla scorta delle carte d'archivio di Santo Stefano, puntualmente citate dall'autore. Purtroppo non è agile ritrovare i documenti segnalati da Nicolai nel fondo archivistico del convento, attualmente custodito presso l'Archivio di Stato di Venezia. Un'inventariazione ulteriore, risalente probabilmente al secolo successivo, ha fatto perdere le tracce della numerazione dei processi citati da Nicolai, senza dimenticare che detto inventario è estremamente approssimativo. La fortuna in qualche caso mi ha assistito e qualche piccola novità documentaria è saltata fuori, ma nulla ahimé che interessi direttamente i dipinti di Corona. de' Cinturati e Cinturate di S. Agostino e di S. Monica sotto l'invocazione di Maria Santissima di Consolazione : e nel primo giorno dell'anno seguente 1582, essendo Priore del Convento il P. M.ro Fra' Raffaello Giordani Veneto, si fece la Processione generale per la Città da detti Fratelli, accompagnati da nostri Religiosi, e con molta pompa e solennità di suonatori di diversi strumenti ».

9 Si ricorda che la Mariegola altro non è che l'insieme delle regole che scandiscono la vita di una confraternita o scuola di devozione. Essa viene normalmente approvata dal Capitolo della chiesa presso cui la confraternita ha sede e pubblicamente registrata dai Provveditori di Comun. Per il documento dell'ottobre 1583 si veda ASVe, Santo Stefano, b. 24, fascicolo segnato " N ${ }^{\circ}$ CCCLXIII », cc. 47r-v - 49r. 
essa compagnia fatto elletione ultimamente, e con l'aiuto di Reverendi Signori et a bossoli, di alcuni officiali come di Guardiano, Avicario, scrivano et di tre altri li quali dal detto corporale hanno facultà di far per esaltatione di detta compagnia alcune dimande alli P.V.M.N. con farle qualche proposta che parerà conveniente $[\ldots]^{\text {I0 }}$.

Purtroppo non conosciamo i nomi dei confratelli ${ }^{\text {II }}$ scelti in seno alla compagnia per avanzare le richieste divenute ormai impellenti al convento : il documento non ne fa menzione alcuna. Piuttosto ragionevoli e ferme al tempo stesso appaiono invece le pretese della confraternita dinanzi ai padri eremitani : innanzitutto la richiesta di attribuzione di un « loco » in chiesa per poter collocare il banco della scuola, suggerendo a siffatto scopo lo spazio esistente in prossimità dell'altare di Santo Stefano, in corrispondenza della colonna più vicina a detto altare. Quanto alle funzioni religiose, i confratelli chiedono che sia celebrata una messa cantata presso l'altare di santa Monica, che si reciti il vespro, che il giorno della festa, ossia la prima domenica dell'avvento, si cantino messa e vespri e che il lunedì a seguire la quarta domenica del mese sia celebrata una messa cantata in onore dei fratelli e sorelle defunti della compagnia. Ma ancora - fatto più interessante - che gli eremitani consegnino al guardiano e al vicario « quelle cose over robbe » di pertinenza della scuola. Solo alle cariche più alte deputate alla gestione della scuola, precisa ancora il documento, spetta in effetti la giurisdizione sulla stampa di libri, la fabbricazione di cinture, le elemosine, i legati e quant'altro. In cambio dell'avvenuto riconoscimento, che si direbbe coincidere con una sorta di emancipazione della scuola dallo stretto controllo dei religiosi, la compagnia si dichiara disponibile a versare la somma di ducati quattro all'anno nelle casse del convento.

Nel 1583 la scuola dei Centurati non possiede dunque un altare di propria pertinenza e si appoggia all'altare dedicato a Santa Monica per la celebrazione delle funzioni religiose. Necessitando anche di un luogo per le proprie riunioni, i Centurati chiedono la possibilità di usufruire del " refettorio quando sarà bisogno di congregarsi, e ordinar le cose che saranno a honor d'Iddio, e

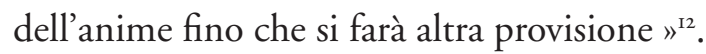

A breve distanza di tempo, i confratelli sono costretti a sottoporre agli Eremitani una nuova serie di richieste ${ }^{\mathrm{r}}$, giacché il documento precedente non ha ricevuto risposta alcuna, quasi non fosse mai stato scritto. Se i toni introduttivi si fanno più morbidi, le pretese sono decisamente più importanti. Vale la pena di sottolineare - ci tornerà utile anche per l'analisi del monocromo e del dipinto - che tra le novità introdotte dal secondo documento compare la richiesta di rimettere ai confratelli l'intera cassa « del seminario et della Compagnia, cioè limosine, legatj, con altre che

\section{Ivi, c. $47 \mathrm{r}$.}

Grazie alla Mariegola conosciamo almeno il nome dei confratelli che ricoprono le cariche di governo della scuola nel 1586 : personaggi su cui sto indagando ma su cui per il momento non posso dire molto. Si veda Gastone Vio, Le Scuole Piccole nella Venezia dei dogi : note d'archivio per la storia delle confraternite veneziane, Costabissara, 2004, p. 320 e per maggior completezza ASVe, Provveditori di Comun, reg. V, c. 433 v. ASVe, Santo Stefano, b. 24, c. $47 \mathrm{v}$.

Si tratta di un altro documento inedito, questa volta non datato, ma plausibilmente di poco successivo al precendente (la grafia è identica). Ivi, cc. 48r-49r. 
potessero avenire per beneficio di detto seminario et di detta compagnia $»^{14}$, insieme al desiderio della scuola di eleggere " un fratino [ossia un novizio] ognj anno, qual sarà eletto dalla compagnia $»^{15}$ " che sia assistente al servitio dell'Altare, dove si comunica le persone, [...] $»^{16}$. Compare inoltre per la prima volta nei documenti la figura del Padre Custode, un eremitano di Santo Stefano appositamente scelto all'interno della comunità dei religiosi per presenziare alle riunioni della confraternita, tenere in ordine l'altare con tutti gli oggetti di pertinenza dello stesso, andar a far la questua in nome della compagnia, confessare i fratelli e le sorelle, etc. ${ }^{17}$ All' uso del refettorio quale luogo di riunione si sostituisce la richiesta che sia messo a disposizione della confraternita " un luogo da servirsi per oratorio ", nonché un " terreno per far due sepolture " nel chiostro del convento $^{18}$. Tra gli effetti di proprietà della scuola, i confratelli reclamano anche " i libri ", parte dei quali essa stessa si è occupata di far stampare a cura di tale mastro Ambrogio ${ }^{19}$. E «Perché bisogna spesso congregarsi a utili della compagnia [...] » e non sono rare le occasioni in cui il Priore del convento o il Maestro dei costumi non esitano a inventarsi scuse su scuse, « pertanto noi volemo liberamente authorità di poter far le congregationi et proponer cose a beneficio del seminario, et a utile della compagnia senza impedimento alcuno $»^{20}$. Le tensioni sono evidentemente all'ordine del giorno tra i confratelli e le alte sfere del convento che continuano con ogni mezzo a cercare di bloccare il tentativo della scuola di rendersi maggiormente autonoma.

I4 Ivi, c. $48 \mathrm{r}$.

I5 Ibid.

I6 Informazioni sui compiti del novizio non esplicitate dal documento fin qui citato si ritrovano invece sulla Mariegola da cui ho estrapolato la seconda parte della citazione. Vedi ASVe, Provveditori di Comun, reg. V, c. 444v. Egli svolgeva dunque per i Centurati le funzioni di un moderno chierichetto. Sul ruolo del Padre Custode si veda il capitolo primo della Mariegola in ASVe, Provveditori di Comun, reg. V : "Matricola della Scola della Beata Vergine della Centura in S. Stefano ", cc. 433v-434r : "Capitolo Primo / Che sia eletto un reverendo padre spirituale dell'ordine di Santo Agostino della Chiesa di Santo Stefano, il quale sia di buona vita, fama, et scientia per benefficio della nostra Compagnia, il quale intervenghi nelle Congregationi, che si faranno, et habbi obligo di confessar tutti li nostri Fratelli, et sorelle, che vorranno confessarsi da lui raccordandogli l'Indulgentie che giornalmente s'acquistano per salute dell'anime loro. / Item sia in obligo al devotissimo Officio come nella Bolla della Santa Felice memoria di PP Gergorio XIII, et di mettere ad ordine l'Altare, della nostra Compagnia, et haver cura di tutte le robbe pertinenti al detto Altare, le quali li debbano essere consignate per Aventario, et così ogni, et qualunque cosa li pervenisse nelle mani di essa Compagnia sia in obligo di consignarle al Mag.co Guardiano, et successori. / Et se occorresse per qualche accidente, che tralasciasse il detto Padre Custode l'officio suo debba, et sia in obligo di consignar tutte le robe, le quali haverà havuto in custodia, consignarle à quello, che li sarà ordinato dal // [c. 434r] Magnifico Guardiano. Sia avvertito nel benedir la Centura, che non riescano scandali, et che li fratelli, et sorelle, che sono ricevuti nella Compagnia farli capaci, et dechiarirli tutte l'immunità, gratie, et privileggi, che li acquistano, tenendo special cura, che la Processione della quarta Domenica sia benissimo regolata, et il tutto sia fatto à laude, e gloria di Sua Divina Maestà, et à benefficio della detta Compagnia, et salute dell'anime di nostri fratelli ». Mi pare importante sottolineare che tra i requisiti essenziali del Padre Custode è richiesta una certa "scientia » : non a caso il primo Padre custode eletto dalla confraternita nel I586 è un baccelliere in teologia, tale fra’ Domenico da Santa Vittoria. Vedi Ivi, c. 433r. ASVe, Santo Stefano, b. 24, c. 48r.

I9 Ivi, c. $48 \mathrm{v}$.

$20 \quad$ Ibid. 
Cosa si può dedurre da questa mole di notizie più o meno scomposte?

La confraternita dei Centurati non è una scuole di devozione qualsiasi, sorta in una delle tante parrocchie di Venezia. Intanto essa nasce e vive in seno a una comunità religiosa e convive dunque con le esigenze dei padri eremitani; in secondo luogo non è affidata esclusivamente alla gestione di laici, comparendo tra le cariche anche il Padre Custode, figura di riferimento in materia di teologia ${ }^{21}$ e pratiche rituali. Infine se l'obiettivo principale dei Centurati resta il soccorso dei " poveri fratelli secondo possibilità ", essi si occupano ugualmente di "statuire, dare ordini, capitolare, et fare altre utilità per la Compagnia dell'Altare, et per il luogo de' novitij, [...] $»^{22}$.

Quanto agli aspetti più propriamente teologico-devozionali, sarà opportuno precisare che la cintura di cui la Scuola si fa in un certo senso depositaria si riferisce a un episodio della vita di Monica, madre di Agostino, divenuto poi esemplare per gli eremitani. Rimasta vedova, la santa donna si rivolge in preghiera alla Vergine, per sapere come Maria avesse vissuto la propria vedovanza e quali abiti sarebbe stato più opportuno indossare. La Vergine le appare allora con indosso una misera veste di colore scurissimo, stretta in vita da una rozza cintura in pelle con tanto di fibia, e ne offre una identica a Monica. La cintura diviene allora prezioso simbolo del legame privilegiato della Vergine con Monica e Agostino, e di lì per transizione con tutto l'ordine degli eremitani ${ }^{23}$.

Ma i confratelli della scuola dei Centurati che in un primo momento fissano la festa della compagnia la prima domenica dell'avvento, nel 1582 decidono che essa avrebbe dovuto celebrarsi piuttosto nel giorno dell'Assunzione della Vergine ${ }^{24}$. Segno di una contaminazione che già alle origini vedeva coinvolto il culto della cintura di santa Monica con quello della cintura che Tommaso riceve dalla Vergine al momento dell'Assunzione, quando l'apostolo si rifiuta di credere che Maria sia stata accolta in cielo anima e corpo ${ }^{25}$. Non stupisce allora che iconograficamente parlando, la Madonna della Cintura ricalchi spesso il modello della Vergine assunta, che per Corona e più in generale per la pittura veneta del XVI secolo vuole innanzitutto dire il capolavoro di Tiziano ai Frari.

2I Vedi in proposito la nota 17.

22 ASVe, Provveditori di comun, reg. V, c. 433r.

23 Su Monica si veda Agostino Trapé, s.v. "Monica, madre di S. Agostino, santa ", in Bibliotheca sanctorum, Roma, 1967, IX, p. 548-562. Per l'iconografia il paragrafo di Angelo Maria Raggi della già menzionata voce, ivi, p. 558-562.

24 ASVe, Provveditori di comun, reg. V, c. 437r : «All'anno 1582 fu posta, et accettata questa Parte dal Capitolo generale della Compagnia, che la principal festa, et solennità della Nostra Confraternita s'havesse à celebrare nel giorno solennissimo dell'Assontione della Madonna di mezzo Agosto per più rispetti, mà particolarmente per utile grande di essa nostra Compagnia; non essendo solenne il giorno della Nostra Protettrice, e Tutrice, e Madre Santa Monica, e per esser noi anche membra, e figli della gloriosa Madre Maria della Consolazione in Bologna [...]».

25 Sulla morte e assunzione di Maria si vedano soprattutto le innumerevoli versioni dell'apocrifo Dormitio Virginis e per fare un po' di chiarezza sulla complessa tradizione testuale a riguardo, l'introduzione di Luigi Moraldi, Apocrifi del Nuovo Testamento, Casale Monferrato, 1994, p. I63-179. 


\section{Questioni materiali}

Il supporto del nostro monocromo è costituito da sei tavole di notevole spessore (all'incirca $5 \mathrm{~cm}$ ) e di larghezza variabile. I bordi laterali sono piuttosto irregolari e il legno presenta numerose abrasioni $^{26}$. All'epoca del ritrovamento, suppongo ${ }^{27}$, i restauratori sono intervenuti sul monocromo limitandosi ad apporre alcune staffe di metallo posizionate orizzontalmente sul retro per tener insieme il tavolato, originariamente inchiodato sull'altare.

La composizione, interamente eseguita a pennello nero e lumeggiature bianche (biacca?), è tracciata su una sottilissima preparazione brunastra a gesso e colla che si rintraccia a fatica in qualche rara zona. Al centro della scena si stagliano imponenti le figure di sant'Agostino con il libro e il pastorale, e di santa Monica con il crocifisso. Entrambi sono raffigurati seduti con lo sguardo rivolto verso la Vergine che ormai non c'è più, ma di cui si intravede il piede sollevato da uno degli angioletti e il drappo finale della veste che un altro angioletto ha preso tra le mani, proprio come nel dipinto su tela. Le altre due figure in secondo piano devono identificarsi plausibilmente con Nicola da Tolentino e Guglielmo. Anche qui come nella versiona definitiva, si intravede il libro di san Nicola, mentre Guglielmo non sembra esibire alcun attributo ${ }^{28}$. Nella porzione alta della tavola compare la folla di angioletti che volteggia tra le nuvole nelle attitudini più disparate. Manca, e vale la pena di notarlo immediatamente, Stefano, la cui presenza certo i religiosi avranno preteso per rendere omaggio al santo titolare della chiesa e del convento. E manca pure la bellissima figura del « fratino » biancovestito, di cui diremo di seguito. Non vi sono dubbi, mi pare, che le due

26 Non sono in grado per il momento di pronunciarmi sull'essenza del legno. Si tratta in effetti di un aspetto fondamentale per stabilire la natura dell'oggetto di fronte a cui ci troviamo. Si ricorda a tal proposito che le tavole su cui si dipingeva all'epoca a Venezia erano quasi esclusivamente in pioppo. Per le questioni materiali sono stata straordinariamente soccorsa da un'abile restauratrice della Soprintendenza per il Polo Museale di Venezia, la dott.ssa Gloria Tranquilli che in questa occasione ringrazio di cuore. Oltre a dimostrare la più totale disponibilità e generosità, il suo intervento e il suo parere sono stati per me preziosissimi e senza di essi non avrei potuto sostenere l'ipotesi che segue.

27 La supposizione è d'obbligo giacché pur esistendo una scheda di restauro, la numero 2509, essa riporta esclusivamente il nome del già menzionato responsabile del cantiere, Francesco Valcanover, la data I97I, e il nome dei restauratori, i fratelli Serafino e Ferruccio Volpin. Essa peraltro non fa menzione del ritrovamento del monocromo. È stata necessaria una lunga ricerca negli archivi della Soprintendenza per ritrovare un negativo con la foto del tavolato al momento del rinvenimento, indicante il numero 26669. Ringrazio moltissimo il dott. Giulio Manieri Elia per avermi messo a disposizione con una velocità insolita per l'amministrazione italiana tale materiale, e le sue assistenti per la paziente ricerca e disponibilità.

28 Mi sono chiesta se gli oggetti che si trovano immediatamente sotto la figura di san Guglielmo appartengano proprio a lui. Si tratta di una corona dorata ornata da alcune pietre preziose e di un altro oggetto metallico non meglio identificabile - forse un brano dell'armatura (un elmo di fattura medievale?) che il santo esibisce in qualche caso, a ricordare il suo passato di condottiero. Va poi precisato che se effettivamente si tratta di un san Guglielmo, l'unico santo con questo nome che abbia a che vedere con la comunità degli Eremitani è in realtà Guglielmo di Malavalle, discepolo di san Bernardo e fondatore dell'ordine dei Guglielmini, aggregatosi a un certo punto con quello degli Eremitani. L'iconografia di quest'ultimo è spesso frutto di contaminazione con il più celebre Guglielmo d'Aquitania, con cui peraltro lo identificano le fonti più antiche. Sui due Guglielmi si veda Rombaut Van Doren, «Guglielmo, monaco a Gellone » e Sergio Mottironi, « Guglielmo il Grande, o di Malavalle ", in Bibliotheca sanctorum, Roma, I966, VII, pp. 467-470 e 47I-473. 

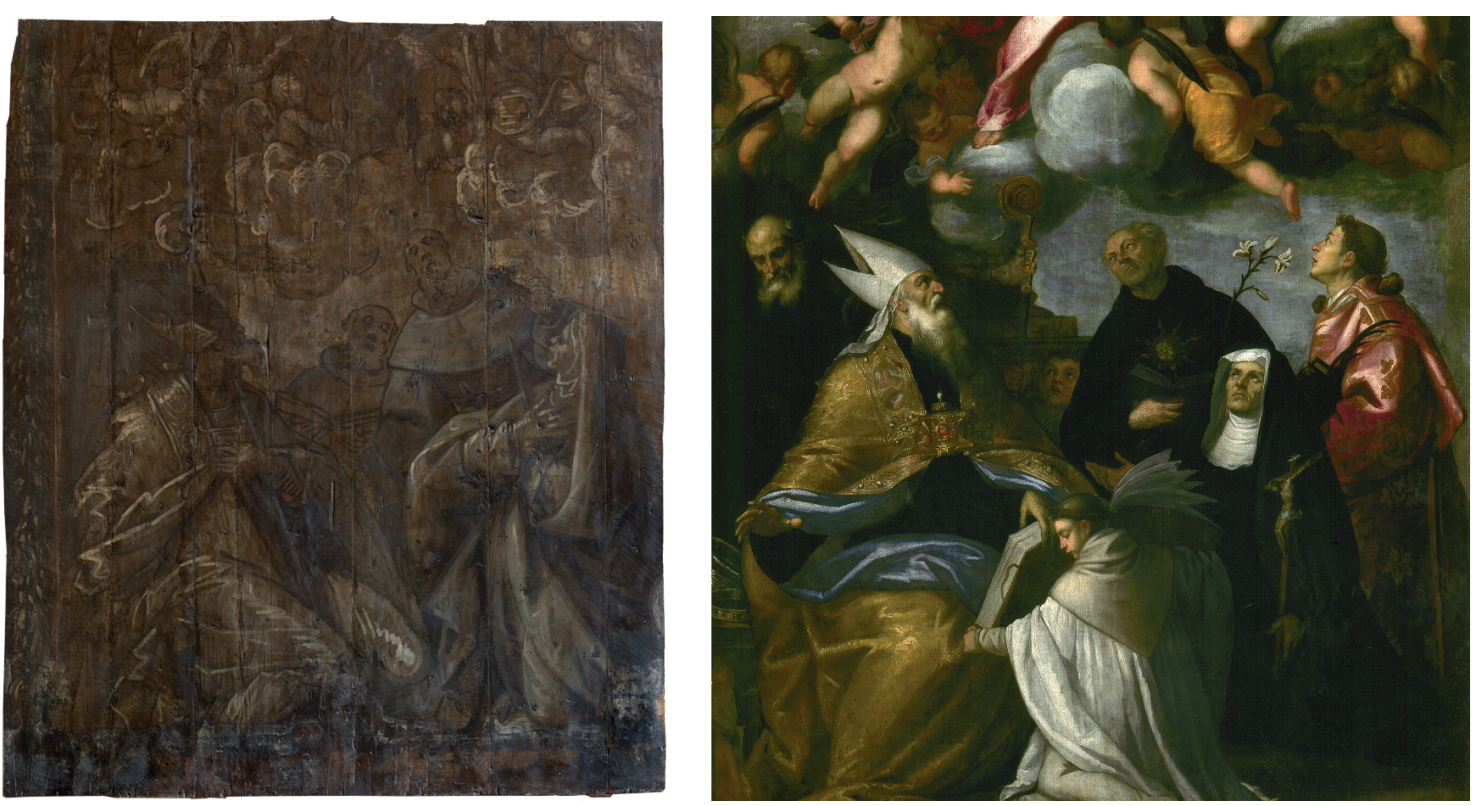

Fig. 4 - Leonardo Corona, Les saints Augustin, Monique, Nicolas de Tolentino et Guillaume d'Aquitaine (détail), I590 et La Vierge à la ceinture avec les saints Augustin, Monique, Nicolas de Tolentino et Guillaume d'Aquitaine (détail), I59I-92, Venise, église de Santo Stefano (C) Cameraphoto, Venise.

opere siano state concepite all'unisono e che la tela costituisca semplicemente l'evoluzione ultima del percorso creativo del pittore, in accordo con le esigenze della committenza (fig. 4).

Per tornare a questioni di ordine materiale, un'altra considerazione mi pare fondamentale a questo punto del discorso : come si è già accennato, Corona ha steso una preparazione sottilissima - qualche millimetro appena - e il tavolato ha un aspetto estremamente grezzo. Sono lasciati a bella vista i nodi del legno, le imperfezioni, le giunzioni fra le tavole, difetti che avrebbero gravemente nuociuto alla buona riuscita di un'opera pittorica su tavola, se di questo si fosse trattato. Manca in sostanza l'operazione prima cui ogni buon pittore dovrebbe attendere quando si avvicina al supporto che accoglierà l'invenzione : la stesura di una preparazione atta a ricevere la pellicola pittorica. Nessun committente avrebbe mai accettato un dipinto il cui supporto lasciasse trasparire i difetti del legno, né tanto meno un pittore raffinato come Corona avrebbe potuto trascurare una tappa fondamentale come la stesura di un'adeguata preparazione. Possiamo quindi escludere che il monocromo costituisca una prima versione del dipinto abbandonata in corso d'opera per ragioni che ignoriamo.

La natura del supporto e ancor di più le dimensioni del monocromo $(324 \times 274 \mathrm{~cm}$ contro i 490 x 260 della tela : la differenza per l'altezza è dettata naturalmente dal fatto che la tavola è stata mutilata nella parte superiore), ci obbligano ugualmente a mettere in dubbio l'ipotesi che possa trattarsi di un bozzetto nel senso stretto del termine. Occupiamoci innanzitutto del supporto. 
La tavola è in effetti un supporto scarsissimamente utilizzato nella seconda metà del Cinquecento a Venezia. L'unico esempio più o meno coevo che mi viene in mente è il bellissimo San Gerolamo di Tiziano (I556-60) alla Pinacoteca di Brera, realizzato per l'altare della famiglia Helman della distrutta chiesa di Santa Maria Nova. In quel caso, la scelta della tavola quale supporto potrebbe esser stata dettata dalle propensioni culturali della committenza : Enrico Helman era un mercante tedesco, originario di Colonia, con affari e famiglia ad Anversa. La scelta di un supporto come la tavola ancora largamente in uso nella cultura nordica fino al XVII secolo - si pensi ad esempio a Rembrandt - potrebbe dipendere da un'apposita richiesta del committente. Nel caso di Corona invece non ci sono dubbi che l'uso della tavola costituisca in qualche modo un'anomalia che non ha apparenti giustificazioni. Peraltro la confraternita dei Centurati non disponeva di particolari ricchezze e non sembra ci siano ragioni particolari per cui abbia potuto pretendere un supporto più costoso e meno pratico, per di più per la realizzazione di un bozzetto.

Inutile poi aggiungere che se di un bozzetto si fosse trattato, esso non dovrebbe possedere le stesse dimensioni (o perfino leggermente superiori) del dipinto su tela : per sua natura il bozzetto è un' « idea ", una " prova " sottoposta alla committenza che magari l'ha pretesa a titolo di verifica preventiva. Non conosco esempi di bozzetti che posseggano le stesse dimensioni del dipinto definitivo, soprattutto quando si tratti di opere tanto imponenti, quali ad esempio una pala d'altare o un grande telero.

Siamo così giunti a una sorta di impasse. Se non si tratta di un bozzetto nel senso stretto del termine e neppure di una prima versione dell'opera successivamente abbandonata, cos'è il nostro monocromo?

\section{Dal « bozzetto » all'opera definitiva : per un'ipotesi storica e materiale}

Il 6 febbraio I590, data della concessione dell'altare alla Scuola dei Centurati, costituisce un utile post quem per il monocromo e la tela di Corona in S. Stefano.

L'evoluzione della carriera di Leonardo Corona, pittore venuto fuori dal nulla e improvvisamente convocato a Palazzo Ducale per decorare insieme a celebri e importanti colleghi il soffitto della Sala del Maggior Consiglio, sfugge ancora profondamente a una comprensione totale. Per la prestigiosa occasione, Corona esegue tre scene a monocromo (!) di straordinario impatto dinamico : Isabella Cornaro consegna al doge Agostino Barbarigo il regno di Cipro, la Battaglia sul lago di Garda e il Restauro dell'istmo di Corinto. Eugenio Manzato ${ }^{29}$ dal canto suo si limita a supporre che se Corona viene ammesso nell'alveo dei pittori del Maggior Consiglio deve aver già dato prova di sé a Venezia, e data dunque a un periodo precedente le Storie della Passione di Cristo nella vicina chiesa di San Zulian ${ }^{30}$. Eppure una lunga e accurata verifica documentaria mi fa supporre che la

\footnotetext{
29 Eugenio Manzato, «Leonardo Corona da Murano », Arte Veneta, XXIV (1970), p. I30.

30 A Corona le fonti attribuiscono l'Ingresso di Cristo a Gerusalemme e Cristo davanti a Caifa. Vittorio Moschini ("Inediti di Palma il Giovane e compagni », Arte Veneta, XII (I958), p. I06) aggiunge anche la Crocifissione,
} 
realizzazione del ciclo di San Zulian, avviata forse alla fine degli anni Settanta, sia poi proseguita almeno fino alla prima metà degli anni Ottanta, ed è dunque più o meno coeva ai dipinti del Maggior Consiglio.

Seguono anni di poco impegno e poi improvvisamente, all'alba dell'ultimo decennio del secolo, Corona si ritrova sommerso di richieste : dalla grande Crocifissione per la Scuola del Santissimo Sacramento di San Fantin, ai dipinti per la chiesa di San Giovanni Elemosinario, alla Crocifissione per l'altare di Marco Barbaro nella chiesa di Santa Maria Formosa. E di lì a breve l'Ecce homo e la Flagellazione per San Giovanni in Bragora, le Storie di San Nicolò per il soffitto della chiesa di San Nicolò de' Mendicoli, quelle della Passione di Cristo per la sala terrena della Scuola de' Picai, e la pala con San Mattia Apostolo in San Bartolomeo ${ }^{31}$, databile al $1595^{32}$ e dunque riconoscibile come una delle ultime opere del pittore, morto di lì a breve il 5 ottobre $1596^{33}$.

Ma il 1590 più di ogni altro momento è un anno di grande, eccessivo impegno, e Corona non ce la fa : finisce per disattendere a uno dei tanti obblighi contratti in buona fede e rischia di pagarne le conseguenze. Il I4 maggio I590 Orazio, fante dei Provveditori di Comun, si presenta dinanzi a " ser Lunardo Corrona Pittor » per intimargli la restituzione della caparra ricevuta dalla Scuola del Santissimo di San Fantin, essendo ormai trascorso « il termine che ha havuto di principiar il quadro $»^{34}$ per la scuola del Santissimo Sacramento di San Fantin. Leonardo dunque non ha neppure cominciato a dipingere l'enorme Crocifissione, commissionatagli evidentemente qualche tempo prima. Di lì a due giorni e probabilmente in risposta a una supplica del pittore, il Provveditore Nicolò Pisani concede a Corona una proroga di sei mesi, entro cui però il pittore dovrà assicurare la consegna del dipinto finito di tutto punto ${ }^{35}$. La vicenda non ha ulteriore seguito, lasciandoci immaginare che tutto si sia concluso per il verso giusto.

Se l'ingiunzione di Nicolò Pisani obbliga Corona a consacrarsi più o meno esclusivamente all'esecuzione della Crocifissione per San Fantin, possiamo ipotizzare che il pittore sia stato costret-

mentre F. Manzato ( Leonardo Corona... », art. cit. nota 29, p. I3I) ritiene che di mano del pittore siano pure il Cristo davanti a Pilato e la Deposizione.

E probabilmente qualche altra opera che per il momento non siamo in grado di datare con certezza. Penso ad esempio alla bellissima pala con Sant'Onofrio, San Rocco e una beata (?), eseguita per l'altare della Scuola dei Tintori in Santa Maria Maggiore (ora a Castelfranco, Duomo, sacrestia) o ancora alle numerose opere perdute e citate dalle fonti. Archivio di San Bartolomeo presso l'Archivio di San Salvador, Atti della Scuola di San Mattia, b. 2 (Scritture diverse), fasc. I (Libro di cassa), c. non numerata, alla data Io marzo I594 (m.v.). ASPV, San Canciano, Registri dei battesimi, matrimoni e morti (25 sett. I564 - 9 giu.I626), b. I, c. non numerata, lettera «L "; e ASVe, Provveditori alla Sanità, Necrologi (1595-96), b. 826, alla data I7 ottobre I596. Vedi V. Sapienza, "Leonardo Corona I552-1596", op. cit. nota 4, p. 198.

34 ASPV, San Fantin, Tomi e atti diversi, b. 32 (Chiesa, sepoltura e scuole), c. 237. Gastone Vio che per primo pubblica il documento lo segnala in San Fantin, Tomo XXXVIII, Chiesa, sacristia e fabrica, c. I47v. Vedi Gastone Vio, "I mistri della chiesa di San Fantin in Venezia ", Arte veneta, XXXI (1977), p. 230. Personalmente ne ho rinvenuto copia in una trascrizione inedita della Mariegola della Scuola : un documento prezioso perché contiene informazioni più dettagliate della Mariegola originale custodita presso la Biblioteca Correr. Il corsivo è mio. Ibid. 
to a trascurare le altre commissioni e magari a lasciare indietro impegni precedentemente contratti, quali ad esempio la realizzazione della pala d'altare per i Centurati - l'assegnazione dell'altare alla confraternita precede di un mese la vicenda di San Fantin.

E se allora il monocromo su tavola fosse per Corona una soluzione provvisoria per soddisfare un altro cliente esigente, evitando di incorrere in ulteriori problemi giudiziari?

L'idea di un decoro provvisorio ci permetterebbe di rispondere ai numerosi interrogativi sollevati fin'ora. La mancanza di un'adeguata preparazione del supporto si giustificherebbe da sé, trattandosi di un'opera destinata a scomparire dietro la tela definitiva. Così la scelta del supporto e le dimensioni : in effetti la tavola sui cui il monocromo viene eseguito dovrebbe semplicemente identificarsi con il tavolato destinato a ricoprire l'altare per potervi fissare la tela definitiva, non esistendo nel Cinquecento i telai moderni. A questo punto, le dimensioni sono per necessità identiche (o quasi) a quelle della pala. Si spiegherebbe così anche la presenza di un elemento nel monocromo, cui fin'ora non si è accennato : la presenza di una cornice di foglie e frutti che corre lungo il lato sinistro del dipinto e che presumibilmente doveva esistere anche sul lato destro ${ }^{36}$.

Lo stato di conservazione del decoro provvisorio si giustificherebbe perfettamente per l'uso, cui il destino l'ha in seguito costretto. Inchiodato contro la parete per fungere da telaio - e non " da rinforzo ", come recita la scheda della Soprintendenza - il monocromo deve essere staccato dal muro in almeno due occasioni, giacché l'altare della Scuola dei Centurati subisce notevoli trasformazioni nel corso dei secoli : la prima volta nel I642, quando viene ridotto in forma di cappella con tanto di soffitto decorato a fresco dal pittore bolognese Antonio Bernardi ${ }^{37}$; la seconda nel secolo successivo a opera di tale Domenico Pirolli, quando è ridotto a più sobria apparenza e assume presumibilmente le sembianze attuali $i^{3}$. Operazioni queste che potrebbero aver facilmente danneggiato la porzione superiore del tavolato, dov'era la Vergine, e che giustificherebbero dunque anche la mutilazione del monocromo, sostituito nella parte superiore da semplici tavole nude (si veda ancora la foto del rinvenimento, fig. 3).

Se così fosse, il dipinto su tavola di Leonardo Corona costituirebbe una testimonianza più che insolita e al tempo stesso preziosissima : da una parte, l'opera altro non sarebbe che un rarissimo tavolato originale risalente alla fine del XVI secolo; essa costituirebbe inoltre una tappa importante dell'evoluzione del percorso creativo del pittore, da un progetto appena abbozzato - e in tal senso possiamo continuare a utilizzare, seppure tra le opportune virgolette, il termine « bozzetto » - fino all'opera definitiva che certamente avrà dovuto tener conto delle esigenze della committenza e di quelle dei padri eremitani ${ }^{39}$.

Se si osserva con attenzione la fotografia del ritrovamento, si noterà che una porzione della cornice è stato segata e riutilizzata per ricoprire una parte superiore del muro dell'altare. Forse quel brano apparteneva all'altro lato del tavolato, anche se l'irregolarità del bordo destro del monocromo è del tutto simile a quella sul lato sinistro. A. Niero, Chiesa di Santo Stefano..., op. cit. nota 3, p. 20.

Ivi, p. 92 e Agostino Nicolai, Memoria manoscritta..., op. cit. nota 7, c. non numerata [2v].

Il monocromo costituisce inoltre l'unica testimonianza certa dell'attività grafica di Corona, da cui si potrebbe cominciare a ragionare per tentare di rintracciare il corpus dei disegni del pittore muranese. 
Se il monocromo diviene la soluzione ideale per una risposta rapida alle esigenze del pittore, sovraccarico di impegni, e della scuola che vuole almeno assaporare il piacere di veder ornato nel più breve tempo possibile il nuovo altare, esso obbliga Corona e i Centurati a un dialogo forzato con i religiosi del convento. Mi pare infatti più che probabile che siano stati proprio questi ultimi a reclamare la presenza del santo titolare, il protomartire Stefano, nell'opera defintiva e a costringere il pittore a ripensare l'insieme della composizione, in un certo senso ai danni della confraternita : non solo Monica perde la centralità che insieme ad Agostino ricopriva nel decoro provvisorio, ma nella tela pare quasi un errore, appiattita com'è nella veste nera e incredibilmente e malamente invecchiata. Ma il riscatto dei Centurati e degli splendidi pennelli di Corona, cui certo i padri non poterono opporsi, è rappresentato dall'introduzione del novizio biancovestito che si tramuta in leggio vivente a sostegno dell'imponente volume di Agostino : il novizio mostra infatti gli stessi tratti fisiognomici seppur più giovani di Stefano, quasi a dire che per raggiungere la perfezione spirituale che permette al protomartire di levare il capo e volgere lo sguardo alla Vergine assunta, essenziale è certo il ruolo svolto dagli eremitani, assistiti però dalla generosità della confraternita impegnata a "proponer cose a beneficio del seminario $[\ldots] »^{40}$. 
